\title{
Biothérapie et odontologie
}

\section{Biotherapy and dental care}

\section{MOTS-CLEFS :}

- Biothérapie, chirurgie orale, maladies inflammatoires, oncologie

\section{KEYWORDS:}

- Biotherapy, oral surgery, inflammatory diseases, oncology

AOS 2013;263:19-31

DOI: $10.1051 / \mathrm{aos} / 2013304$

(C) EDP Sciences 2013

\section{Résumé}

Les biothérapies correspondent ò l'utilisation d'une molécule, de cellules, voire de tissus, à des fins thérapeutiques. L'un des principaux outils actuels des biothérapies est représenté par les anticorps monoclonaux et leurs dérivés. Ils sont devenus, notamment grâce ò̀ une remarquable ingénierie moléculaire, des outils thérapeutiques de premier plan dans des domaines cliniques très divers, notamment la rhumatologie, la cancérologie, l'hématologie et l'hépato-gastro-entérologie. À l'image des anti-TNF $\alpha$ et des anti-IL-1, les biothérapies sont des immunothérapies ciblées qui ont des applications particulièrement intéressantes dans les rhumatismes inflammatoires et les maladies auto-immunes.

Leur production et leur utilisation à grande échelle ont été possibles grâce aux progrès considérables du développement industriel des biotechnologies. Or, la réalisation d'une intervention chirurgicale lors d'un traitement par biothérapies, notamment les anti-TNF $\alpha$, peut, théoriquement, conduire ò un risque d'infections per- ou postopératoires augmenté.

Les perspectives sont immenses en ce qui concerne les biothérapies. Reste ò définir les bonnes indications et les stratégies thérapeutiques les plus pertinentes mais aussi la tolérance au long et très long cours de ces nouvelles molécules. Leur utilisation est de plus en plus fréquente et il convient de bien connaître les précautions à prendre pour ces patients lors de leur prise en charge odontologique.

\section{Abstract}

Biotherapies is the use of a molecule, cells, even tissue for therapeutic means. Currently, one of the other major bio-therapeutic tools are monoclonal antibodies and their by-products. They came into being notably thanks to remarkable molecular engineering, a cornerstone for therapeutic tools in a wide range of clinical domains such as oncology, hematology and gastroenterology. As with the anti-TNF $\alpha$ and the anti-IL-1 biotherapies are specific immunotherapies which have the particularly interesting applications for inflammatory rheumatism and auto-immune diseases.

Their production and use on a large scale have been made possible thanks to enormous progress in the biotechnology industry. However, carrying out surgery during anti-TNF $\alpha$ treatment can, theoretically, result in a higher risk of infections in pre or post-surgery.

The prospects for biotherapies are huge. Not only are the correct indications to be defined along with the most pertinent therapeutic strategy to follow but also the tolerance of long to very long use of these new molecules. Their use is becoming more and more frequent and it is necessary to know what precautions to take for one's patients during odontological care.

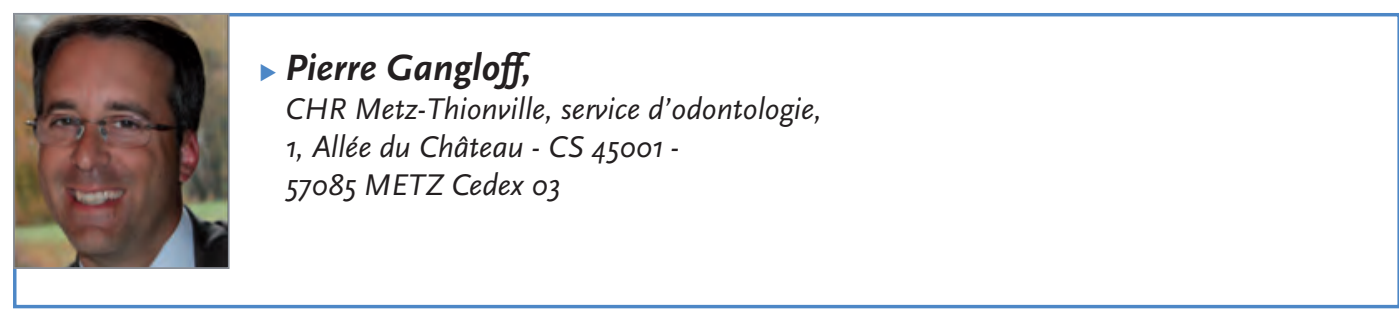




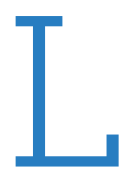
e terme « biothérapies » recouvre un ensemble de traitements d'origine immunologique destinés principalement à agir contre des molécules du système immunitaire impliquées dans la physiopathologie de maladies inflammatoires rhumatologiques (polyarthrite rhumatoïde : PR, spondylarthrite ankylosante, rhumatisme psoriasique), digestives (maladies inflammatoires chroniques de l'intestin: MICI), neurologiques (sclérose en plaque), cutanées (psoriasis). L'utilisation des biothérapies en rhumatologie a profondément modifié la prise en charge des patients porteurs de rhumatismes inflammatoires chroniques. La deuxième indication majeure des biothérapies se retrouve dans le domaine de la cancérologie où elles sont utilisées comme antinéoplasiques

L'un des principaux outils actuels des biothérapies est représenté par les anticorps monoclonaux (AcM) et leurs dérivés. Les AcM sont devenus, notamment grâce à une remarquable ingénierie moléculaire, des outils thérapeutiques de premier plan dans des domaines cliniques très divers.

Le succès de cette première génération d'anticorps a lancé de nouveaux défis comme la conception d'anticorps aux activités fonctionnelles optimisées et aux effets secondaires mieux contrôlés, ou la production à moindre coût. Une nouvelle génération d’anticorps est en train d'apparaître, et le futur se profile déjà à l'horizon : approches oligoclonales fondées sur l'utilisation de cocktails de différents anticorps monoclonaux, sélection des patients éligibles, production en masse à des coûts moindres...

\section{QUELLES BIOTHÉRAPIES POUR QUELLES INDICATIONS ?}

Avec le développement de nouveaux traitements ciblés dans la PR ou les MICI, le choix du traitement passe par un ensemble de critères généraux rigoureux et de critères spécifiques au malade traité (Tableau IA et IB). Les trois critères généraux sont :

I les indications selon le respect de l'AMM;

$\checkmark$ les recommandations des agences ou autorités de santé;

I le choix personnel du praticien et du service hospitalier, car ces biothérapies sont toutes de prescription hospitalière initiale, a fortiori en cas d'administration intraveineuse.

Sont donc à évaluer :

I les caractéristiques du patient (âge, sexe, choix de procréation, état veineux, contexte culturel, compliance et préférences);

I ses comorbidités;

1 l'activité de la maladie selon des critères validés comme le DAS 28-VS (Disease Activity Score);

I à quoi s'ajoutent d'autres caractéristiques de sévérité de la PR: caractère érosif, évolutivité structurale, présence de biomarqueurs (facteur rhumatoïde et anti-

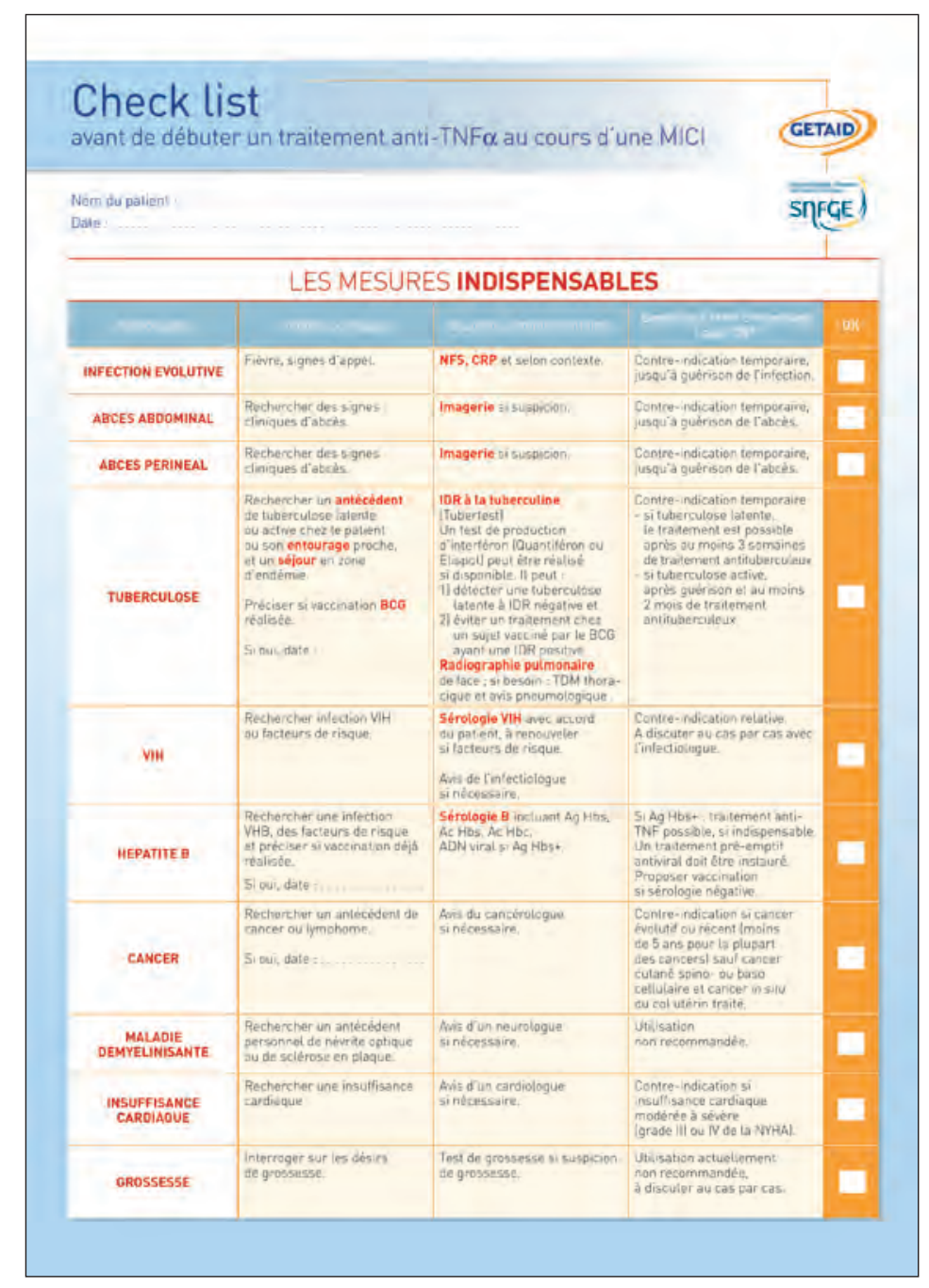

corps antipeptides citrullinés). Le choix va également dépendre des échecs aux thérapeutiques antérieures, définissant ainsi des choix de biothérapies.

Le rhumatologue a pu et doit choisir parmi plusieurs types de traitements disponibles chronologiquement: agents anti-TNF (infliximab, adalimumab), anticorps (Ac) anti-IL-1ra (anakinra), Ac anti-CD20 (rituximab), régulateur de la costimulation (abatacept) et Ac anti-IL-6R (tocilizumab). Leurs caractéristiques, leurs voie et rythme d'administration sont à prendre en compte, surtout les données de pharmacovigilance, le recul disponible améliorant la sécurité d’emploi. Comme pour un antibiotique, on peut ainsi proposer de commencer par le plus ancien, en l'absence d'informations en termes de différences d'efficacité selon la phase évolutive de la PR. Cette attitude est conforme au principe de précaution.

Si l'on souhaite sortir d'un schéma dicté par lordre d'apparition chronologique des biothérapies, on peut faire appel, en première ligne, à un schéma basé sur la physiopathologie actuelle de la PR et distinguer la PR débutante, au cours de laquelle la réaction immunologique $\mathrm{B} / \mathrm{T}$ à un ou des antigènes est au premier plan, et les $P R$ anciennes, pour lesquelles une réaction inflammatoire chronique non spécifique entretient la maladie articulaire. Dans les PR débutantes avec taux 


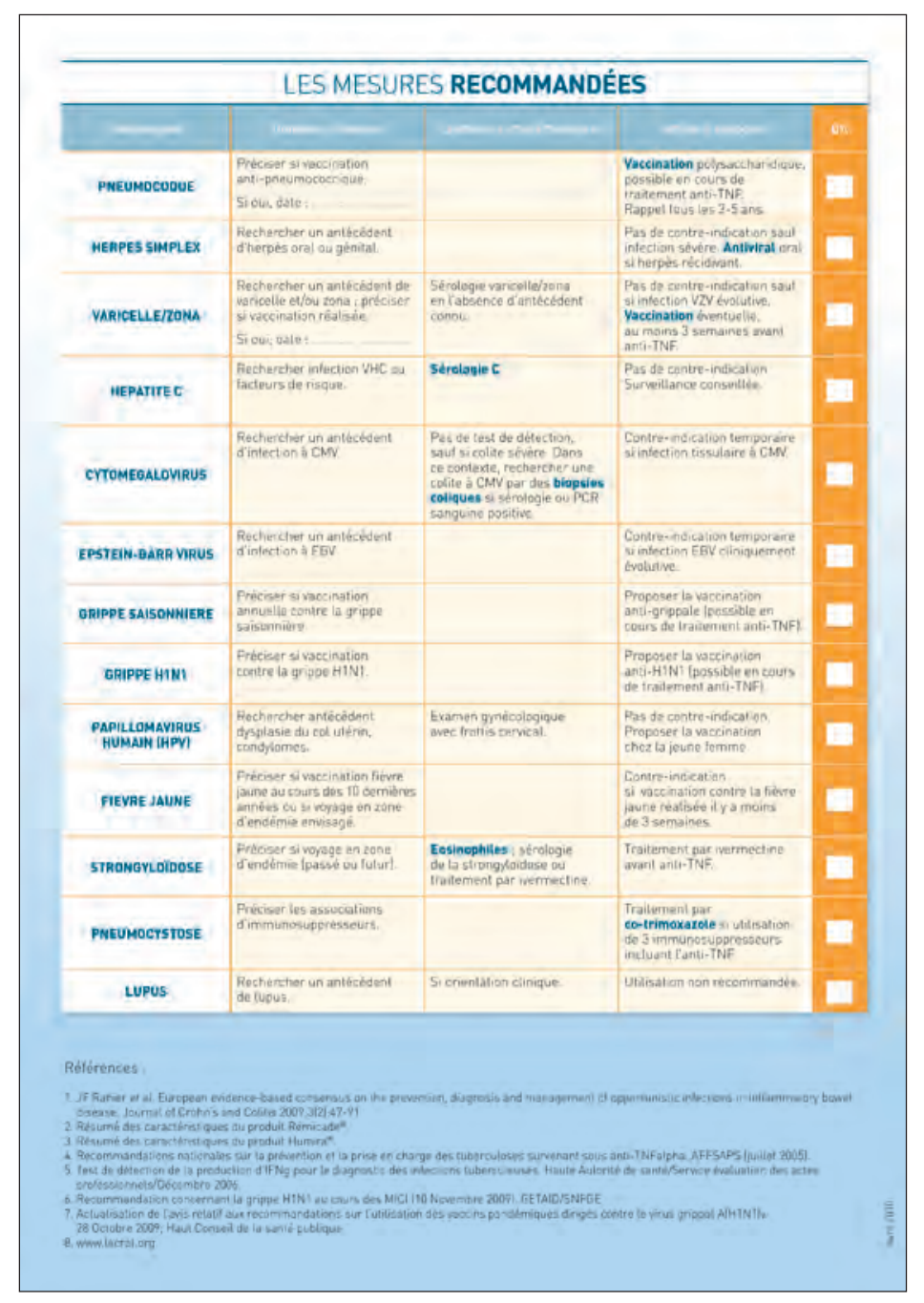

Tableau IA et IB :

Checklist avant de débuter un traitement anti-TNF au cours d'une maladie chronique inflammatoire de l'intestin (disponible en intégralité sur les sites de la SNFGE et du GETAID)

élevés d'auto-anticorps (facteur rhumatoïde, ACPA...) et/ou signes extra-articulaires, une biothérapie anti-B (CD20) serait instituée en priorité. Les PR avec taux modéré d'auto-anticorps ou séronégatives relèveraient en premier lieu d'un inhibiteur des co-signaux d'activation (CTLA4-Ig abatacept). Les PR anciennes érosives seraient candidates à un traitement anti-inflammatoire puissant délivré par les agents anti-TNFa ou un Ac anti-IL-6R (tocilizumab). Un tel schéma demande à être affiné par des études contrôlées comparant les différentes biothérapies entre elles, dans des $\mathrm{PR}$ récentes et dans des $\mathrm{PR}$ anciennes, avec un produit de référence qui serait non pas le méthotrexate, mais un agent antiTNFa. L'AMM de ces produits propose la prescription d'une biothérapie d'emblée en cas de forme débutante "active et sévère».

Il n'y a pas actuellement de biomarqueurs susceptibles d'aider au choix. On pourrait imaginer aussi que des données de pharmacogénétique puissent à terme être disponibles. Ces choix vont encore se compliquer avec l'arrivée d'autres agents anti-TNF. Il faudrait mettre en œuvre des essais «face à face» de deux biothérapies, dont un agent anti-TNF de référence, et des essais pragmatiques comparant les biothérapies entre elles, par exemple en seconde ligne.

\section{LES PRODUITS DISPONIBLES}

- Les anti-TNFa ont pour but de bloquer le TNFa, cytokine pro-inflammatoire. Trois molécules dominent actuellement le marché. Deux sont des AcM : l'adalimumab (Humira ${ }^{\circledR}$ ) est un anticorps monoclonal humain, tandis que l'infliximab (Rémicade ${ }^{\circledR}$ ) est un anticorps monoclonal chimérique (comportant des séquences humaines et murines). La troisième - étanercept $\left(\right.$ Enbrel $\left.^{\circledR}\right)$ - est un analogue d'un récepteur soluble du TNFa (on parle aussi de protéine de fusion). Lefficacité de ces trois molécules a été démontrée sur le plan clinique, biologique et sur la qualité de vie, aussi bien dans la polyarthrite rhumatoïde (PR) que dans la spondylarthrite ankylosante (SA) et le rhumatisme psoriasique (RP). Une efficacité structurale (réduction de la progression des lésions radiologiques) a de plus été clairement établie dans la PR et à un moindre degré dans le rhumatisme psoriasique. Cependant, $30 \%$ des patients traités par anti-TNFa pour une PR sont non répondeurs [1]. Les facteurs prédictifs de réponse au traitement ne sont pas clairement identifiés et il reste difficile de cibler les patients répondeurs, qu'il s'agisse de la PR, de la SA ou du RP. De façon générale, on pense qu'une maladie récente et la présence d'une inflammation importante constituent des facteurs de réponse au traitement à trois mois. Pour autant, certains patients atteints de PR ou spondylarthrite depuis plusieurs années et sans syndrome inflammatoire important répondent de façon spectaculaire aux anti-TNFa.

I Lanakinra (Kineret ${ }^{\circledR}$ ) est un inhibiteur de l'interleukine 1 . Son efficacité est modérée par rapport à celle des anti-TNFa [2].

I D’autres biothérapies agissent en induisant une déplétion d'une population cellulaire impliquée dans le processus pathologique. Ainsi, le rituximab (Mabthera $\left.{ }^{\circledR}\right), A c M$ chimérique déjà utilisé en hématologie dans le traitement des lymphomes, cible le CD-20, molécule exprimée par les lymphocytes B. Labatacept $\left(\right.$ Orencia $\left.^{\circledR}\right)$ est une protéine de fusion inhibant l'activation des lymphocytes T. Ces deux médicaments ont démontré leur efficacité dans la PR en association au méthotrexate (MTX), mais on ne dispose pas d'études les comparants directement aux anti-TNFa.

I Un nouvel AcM anti-interleukine 6, le tocilizumab (Roactemra ${ }^{\circledast}$ ), vient d'obtenir une AMM européenne dans le traitement de la PR.

Le premier AcM mis sur le marché (en 1986) a été le muromonab-CD3 (Orthoclone OKT3 ${ }^{\circledR}$ ), un anticorps de souris dirigé contre la molécule $\mathrm{CD} 3$, exprimée par les lymphocytes $\mathrm{T}$, pour la prévention du rejet aigu des allogreffes rénales, celui-ci a été retiré du marché depuis. Ce n'est qu'en 1994 qu'un second AcM, chimérique, l'abciximab (Reopro ${ }^{\varpi}$ ), dirigé contre la molécule 
GPIIb/IIIa, a obtenu également une autorisation de mise sur le marché (AMM) pour la prévention de la formation de thrombus à la suite d'opérations cardiovasculaires. Le premier AcM humanisé, le daclizumab (Zenapax $\left.{ }^{\circledast}\right)$, dirigé contre la chaîne alpha du récepteur de l'IL-2 (CD25), pour la prévention des rejets aigus des allogreffes rénales, a été mis sur le marché en 1997. Enfin, le premier AcM complètement humain à obtenir une AMM fut l'adalimumab (Humira ${ }^{\circledR}$ ), en 2002, dirigé contre le TNFa et utilisé pour le traitement de la polyarthrite rhumatoïde (tableau II).

En 2008, 22 AcM avaient déjà une AMM de la FDA (Food and Drug Administration) aux États-Unis [2] (tableau III) mais certains ont été retirés du marché depuis. Quatre d'entre eux sont des anticorps ou des fragments d'anticorps de souris, 5 des anticorps chimériques, 11 des anticorps humanisés et 2 des anticorps complètement humains. Enfin, deux autres anticorps humains [zanolimumab (anti-CD4) et ofatumumab (anti-CD20)] ont reçu un statut de médicament orphelin. En 2012, en France, 26 AcM sont commercialisés. Les AcM ont tous un nom générique contenant un préfixe choisi par les inventeurs ou développeurs et un suffixe, «mab» (pour monoclonal antibody). Ce nom générique contient aussi des radicaux définissant la nature moléculaire de l'anticorps (o pour souris, xi pour chimérique, zu pour humanisé, u pour humain...), ainsi que la cible initiale de l'anticorps (li, pour système immunitaire, tu pour tumeur, ba pour bactérie, ki pour interleukine, vi pour virus...). Par exemple, le nom « rituximab» $\left(\right.$ Mabthera $^{\circledR}$ ) désigne un anticorps monoclonal (mab), chimérique (xi), et dirigé contre une molécule (CD20) exprimée à la surface de cellules tumorales $(\mathrm{tu})$.

À ce jour, 26 anticorps monoclonaux sont disponibles sur le marché français et plus de 200 sont évalués dans des essais cliniques (Tableau IV).

Les AcM à usage thérapeutique actuellement sur le marché ont différents modes d'action, parfois non mutuellement exclusifs :

I neutralisation de l'action d'une molécule soluble pro-inflammatoire comme le TNFa [infliximab (Remicade ${ }^{\circledast}$ ) et adalimumab]. La neutralisation du TNFa soluble est également obtenue avec la protéine de fusion étanercept. Des différences d'action semblent cependant exister puisque l'on soupçonne que les différences de réponse observées chez certains patients traités par les anticorps anti-TNFa ou par létanercept pourraient être liées à la capacité des anticorps à cibler également le TNF $\alpha$ membranaire. Il est à noter que la neutralisation d'une autre molécule pro-inflammatoire, l'IL-1a, est obtenue en utilisant une protéine recombinante, l'IL-1-RA, antagoniste du récepteur de l'IL-1a;

I neutralisation d'un facteur soluble de croissance de l'endothélium vasculaire (VEGF), afin de prévenir la néo-angiogenèse tumorale [bevacizumab $\left(\right.$ Avastin $\left.^{\circledast}\right)$ ] ;

॥ inhibition de la migration cellulaire par blocage de l'adhérence cellulaire [natalizumab (Tysabri ${ }^{\circledR}$ )] ;

I blocage par compétition de l'inhibition de l'activation de molécules de costimulation [abatacept (Orencia ${ }^{\circledR}$ )].

\begin{tabular}{|c|c|c|c|c|c|c|}
\hline Molécule & Mode d'action & Indications AMM & Indications en évaluation & Dose & $\begin{array}{c}\text { Voie } \\
\text { d'administration }\end{array}$ & $\begin{array}{l}\text { Associations } \\
\text { thérapeutiques }\end{array}$ \\
\hline $\begin{array}{l}\text { Infliximab } \\
\text { (Rémicade }^{0} \text { ) }\end{array}$ & $\begin{array}{l}\text { Ac monoclonal (IgGl) } \\
\text { chimérique anti-TNF }\end{array}$ & $\begin{array}{l}\text { PR } \\
\text { Spondylarthrites } \\
\text { Rhum pso } \\
\text { Crohn } \\
\end{array}$ & $\begin{array}{l}\text { Uvéites } \\
\text { Vascularites } \\
\text { Sarcoïdose } \\
\text { Maladies auto-immunes }\end{array}$ & $\begin{array}{c}3 \text { à } 5 \mathrm{mg} / \mathrm{kg} \\
12 \mathrm{mois}\end{array}$ & IV & $\begin{array}{l}\text { Méthotrexate } \\
\text { (recommandée) }\end{array}$ \\
\hline $\begin{array}{l}\text { Adalimumab } \\
\text { (Humira }^{\circ} \text { ) }\end{array}$ & $\begin{array}{l}\text { Ac monoclonal ( } \mathrm{IgGl}) \\
\text { humain anti-TNF }\end{array}$ & PR & $\begin{array}{l}\text { Rhum psoriasique } \\
\text { spondylarthropathies }\end{array}$ & $\begin{array}{c}40 \mathrm{mg} / 2 \mathrm{sem} \\
\text { ou toutes les } \\
\text { semaines }\end{array}$ & SC & Méthotrexate (possible) \\
\hline $\begin{array}{l}\text { Etanercept } \\
\left(\text { Enbrel }^{\circ}\right)\end{array}$ & $\begin{array}{l}\text { Protéine de fusion } \\
\text { (TNFR2-Fc) anti-TNF }\end{array}$ & $\begin{array}{l}\text { PR } \\
\text { AJI } \\
\text { Rhum psoriasique } \\
\text { Spondylarthrite } \\
\end{array}$ & $\begin{array}{l}\text { Uvéite } \\
\text { Vascularites } \\
\text { Sarcoïdose } \\
\text { Mal autoimmunes }\end{array}$ & $2 \times 25 \mathrm{mg} / \mathrm{sem}$ & SC & $\begin{array}{l}\text { Méthotrexate } \\
\text { (possible) }\end{array}$ \\
\hline $\begin{array}{l}\text { Anakinra } \\
\left(\text { Kineret }^{\circ}\right)\end{array}$ & $\begin{array}{l}\text { Protéine recombinante } \\
\text { (IL1-Ra) anti-IL-1 }\end{array}$ & PR & $\begin{array}{l}\text { AJI } \\
\text { Maladie périodique et autres } \\
\text { fièvres périodiques } \\
\text { héréditaires }\end{array}$ & $100 \mathrm{mg} / \mathrm{j}$ & SC & $\begin{array}{l}\text { Méthotrexate } \\
\text { (recommandée) }\end{array}$ \\
\hline $\begin{array}{l}\text { Rituximab } \\
\left.\text { (Mabthera }^{\circ}\right)\end{array}$ & $\begin{array}{l}\text { Ac monoclonal (IgG1) } \\
\text { chimérique anti-CD20 } \\
\text { des LB }\end{array}$ & Lymphomes B & $\begin{array}{l}\text { PR } \\
\text { Lupus } \\
\text { Cytopénie } \\
\text { Cryoglobulinémie } \\
\text { Mal auto-immunes }\end{array}$ & A préciser & IV & A préciser \\
\hline Abatacept & $\begin{array}{l}\text { Protéine de fusion } \\
\text { (CTLA4-Ig) } \\
\text { inhibitrice de la } \\
\text { costimulation des LT }\end{array}$ & Non & $\begin{array}{l}\text { PR } \\
\text { AJI } \\
\text { Lupus } \\
\text { Mal. autoimmunes }\end{array}$ & A préciser & IV & A préciser \\
\hline
\end{tabular}

Tableau II :

Principales biothérapies commercialisées ou en cours de développement dans les maladies inflammatoires en France 
Un AcM anti-CTLA-4 est actuellement en cours d'essais cliniques;

I blocage par compétition de récepteurs membranaires de facteurs de croissance comme le récepteur de l'epidermal growth factor (EGF-R) [cétuximab (Erbitux ${ }^{\circledast}$ ), panitumumab $\left(\right.$ Vectibix $\left.^{\circledast}\right)$ ], ou le récepteur de haute affinité de l'interleukine-2 (IL-2) [daclizumab $\left(\right.$ Zenapax $\left.^{\circledR}\right)$ et basiliximab (Simulect $\left.{ }^{\circledR}\right)$, anti-CD25]. Il est à noter que le blocage de l'activation spontanée de l'un des récepteurs de l'EGF, sans ligand connu (HER2/Neu ou ErbB2), dont le gène est amplifié dans environ $20 \%$ des tumeurs du sein, est obtenu avec un AcM [trastuzumab (Herceptin $\left.{ }^{\circledast}\right)$;

I fixation à une molécule membranaire [rituximab $\left(\right.$ Mabthera $\left.^{\circledast}\right)$;

I inhibition de l'entrée d'un virus dans les cellules cibles (virus respiratoire syncitial) [palivizumab (Synagis $\left.\left.{ }^{\circledast}\right)\right]$;

I activation de mécanismes effecteurs : fixation du C1q (premier composant de la voie classique du complément), conduisant à la lyse des cellules cibles (CDC ou complement dependent cytotoxicity) et/ou fixation aux RFcg exprimés à la surface de cellules de l'immunité, conduisant également à une lyse des cellules cibles (ADCC ou antibody dependent cell cytotoxicity) ou à leur phagocytose. Chez l'homme,
l'ADCC, la phagocytose et la CDC sont des fonctions effectrices exercées par les anticorps d'isotype IgG1 et IgG3. C'est pour cette raison que la plupart des anticorps sur le marché, qu'ils soient chimériques, humanisés ou humains sont des IgG1. Il existe seulement sur le marché deux anticorps ayant des Fc appartenant à une autre sous-classe humaine : le gemtuzumab (IgG4) (Mylotarg ${ }^{\circledR}$ ) et le panitumumab (IgG2) (Vectibix ${ }^{\circledR}$ ). L’objectif ouvertement recherché est que ces anticorps n'engagent pas les mécanismes effecteurs de l'immunité.

\section{LES INDICATIONS}

Les indications des principales biothérapies ainsi que le mode d'administration sont résumés dans le tableau II. Leur prescription est réservée aux médecins spécialistes, selon les cas : rhumatologue, médecin interniste, pédiatre, dermatologue, gastroentérologue, oncologue, hématologue.

\section{Dans la polyarthrite rhumatoïde (PR)}

Rappelons que le traitement de fond de première intention, une fois le diagnostic de PR affirmé, est toujours le MTX (sauf contre-indication).

\section{Anticorps monoclonaux à usage thérapeutique sur le marché (FDA) (décembre 2008)}

\begin{tabular}{|c|c|c|c|c|c|}
\hline Nom & Type & Date & Isotype & Cible & Indication \\
\hline Muromonab (Orthaclone 0 OKT $3^{\circ}$ ) & Souris & 1986 & $\lg G 2 a$ & $\mathrm{CD} 3$ & Prévention de rejets aigus d'allogreffes \\
\hline Abciximab (Reopro $\left.{ }^{5}\right)$ & Chimérique & 1994 & Fab & GPIIb/IIla & Prévention des thrombus post-chirurgicaux \\
\hline Capromab pendetide & Souris & 1996 & Fab & Prostate Ag & Imagerie (cancer prostate) \\
\hline Daclizumab (Zenapax ${ }^{8}$ ) & Humanisé & 1997 & IgG1 & $\mathrm{CD} 25$ & Prévention de rejets aigus d'allogreffes \\
\hline Rituximab (Mabthera" ${ }^{2}$ ) & Chimérique & 1997 & $\lg \mathrm{G1}$ & $\mathrm{CO} 20$ & LNH", Arthrite rhumatoïde \\
\hline Basiliximab (Simulectß) & Chimérique & 1998 & IgG1 & $\mathrm{CD} 25$ & Prévention de rejets aigus d'allogreffes \\
\hline Palivizumab (Synagis $^{8}$ ) & Humanisé & 1998 & IgG1 & RSV $^{\text {nt }}$ & Infection à RSV \\
\hline Trastuzumab (Herceptin"') & Humanisé & 1998 & $\lg G 1$ & HER2/neu & Cancer du sein métastatique \\
\hline Infliximab (Remicade ${ }^{a i}$ ) & Chimérique & 1998 & IgG1 & TNF $\alpha$ & Arthrite rhumatoïde, maladie de Crohn \\
\hline Gemtuzumab-ozogamicin (Mylotarg ${ }^{\infty}$ ) & Humanisé & 2000 & $\lg \mathrm{G} 4$ & $\mathrm{CD} 33$ & Leucémie myèloïde aiguẻ \\
\hline Alemtuzumab (Mabcampath ${ }^{3}$ ) & Humanisé & 2001 & IgG1 & $\operatorname{CD} 52$ & Leucémie lymphoïde chronique B (LLC-B) \\
\hline Adalimumab (Humira ${ }^{a}$ ) & Humain & 2002 & $\lg 61$ & INF $\alpha$ & Arthrite rhumatoïde \\
\hline${ }^{90} \gamma$-lbritumomab tiuxétan (Zevalin") & Souris & 2002 & $\lg G 1$ & $\mathrm{CO} 20$ & $\mathrm{LNH}^{\circ}$ \\
\hline omalizumab (Xolair ${ }^{b}$ ) & Humanisé & 2003 & IgG1 & $\lg \mathrm{E}(\mathrm{Fc})$ & Asthme allergique \\
\hline Efalizumab (Raptiva" & Humanisé & 2003 & $\operatorname{lgG1}$ & CD11a & Psoriasis \\
\hline 1311-Tositumomab & Souris & 2003 & $\lg G 2 a$ & $\mathrm{CD} 20$ & LNH \\
\hline Cétuximab (Erbitux") & chimérique & 2004 & $\lg G 1$ & EGF-R & Cancer colorectal Méta. \\
\hline Bevacizumab (Avastin ${ }^{{ }^{2}}$ ) & Humanisé & 2004 & $\lg G 1$ & VEGF & Cancer colorectal \\
\hline Natalizumab (Tysabiri") & Humanisé & 2004 & $\lg G 4$ & $\alpha 4$ & Sclérose en plaques \\
\hline Zanolimumab & Humain & 2005 & $\lg \mathrm{G} 1$ & $\mathrm{CD} 4$ & Lymphome cutané $\mathrm{T}$ \\
\hline Ofatumumab ${ }^{--}$ & Humain & 2005 & IgG1 & $\mathrm{CD} 20$ & LNH, LLC-B \\
\hline Ranibizumab (tucentis ${ }^{\mathrm{min}}$ ) & Humanisé & 2006 & $\mathrm{Fab}^{\prime}$ & VEGF & Dégénérescence maculaire liée à l'âge \\
\hline Panitumumab (Panitumumab" ${ }^{\infty}$, Vectibix ${ }^{8}$ ) & Humain & 2006 & $\lg G 2$ & EGF-R & Cancer colorectal \\
\hline Eculizumab (Soliris ${ }^{8}$ ) & Humanisé & 2007 & IgG1 & C5 & $\mathrm{HPN}^{\text {"*a* }}$ \\
\hline
\end{tabular}




\begin{tabular}{|c|c|c|c|}
\hline Anticorps monoclonal & Nom commercial & Spécialité & Indications \\
\hline Abatecept & ORENCIA & Rhumatologie & Immunosuppresseurs \\
\hline Abciximab & REOPRO & Cardiologie & Prévention des thromboses post chirurgicales \\
\hline \multirow{2}{*}{ Adalimumab } & \multirow{2}{*}{ HUMIRA } & HGE & Maladies inflammatoires de l'intestin \\
\hline & & Rhumatologie & Immunosuppresseurs \\
\hline Alemtuzumab & MABCAMPATH & Hématologie & Leucémie lymphoïde chronique B \\
\hline Basiliximab & SIMULECT & Cancérologie & Immunosuppresseurs sélectifs \\
\hline Bélatacept & NULOJIX & Cancérologie & Immunosuppresseurs sélectifs \\
\hline \multirow{2}{*}{ Bevacizumab } & \multirow{2}{*}{ AVASTIN } & Cancérologie & Antinéoplasiques \\
\hline & & Hématologie & Antinéoplasiques \\
\hline Canakinumab & ILARIS & Cancérologie & Immunosuppresseurs sélectifs \\
\hline Certolizumab & CIMZIA & Rhumatologie & Immunosuppresseurs \\
\hline \multirow{2}{*}{ Cétuximab } & \multirow{2}{*}{ ERBITUX } & Cancérologie & Antinéoplasiques \\
\hline & & Hématologie & Antinéoplasiques \\
\hline Daclizumab & ZENAPAX & Cancérologie & Immunosuppresseurs sélectifs \\
\hline \multirow{2}{*}{ Denosumab } & \multirow{2}{*}{ PROLIA } & Cancérologie & $\begin{array}{l}\text { Inhibiteur du récepteur RANK des } \\
\text { ostéoclastes }\end{array}$ \\
\hline & & Rhumatologie & $\begin{array}{l}\text { Inhibiteur du récepteur RANK des } \\
\text { ostéoclastes }\end{array}$ \\
\hline Eculizumab & SOLIRIS & Hématologie & Hémoglobulinurie paroxystique nocturne \\
\hline Gemtuzumab-ozogamicin & MYLOTARG & Hématologie & Leucémie myéloïde aiguë \\
\hline \multirow{2}{*}{ Ibritumomab tiuxétan } & \multirow{2}{*}{ ZEVALIN } & Cancérologie & Antinéoplasiques \\
\hline & & Hématologie & Antinéoplasiques \\
\hline \multirow{2}{*}{ Infliximab } & \multirow{2}{*}{ REMICADE } & HGE & Maladies inflammatoires de l'intestin \\
\hline & & Rhumatologie & Immunosuppresseurs \\
\hline \multirow{2}{*}{ Ipilimumab } & \multirow{2}{*}{ YERVOY } & Cancérologie & Antinéoplasiques \\
\hline & & Hématologie & Antinéoplasiques \\
\hline Natalizumab & TYSABRI & Neurologie & Sclérose en plaque \\
\hline \multirow{2}{*}{ Ofatumumab } & \multirow{2}{*}{ ARZERRA } & Cancérologie & Antinéoplasiques \\
\hline & & Hématologie & Antinéoplasiques \\
\hline Omalizumab & XOLAIR & Pneumologie & Asthme allergique \\
\hline Palivizumab & SYNAGIS & $\begin{array}{l}\text { Infection ò virus } \\
\text { respiratoire } \\
\text { syncytial }\end{array}$ & Pneumologie \\
\hline \multirow{2}{*}{ Panitumumab } & \multirow{2}{*}{ VECTIBIX } & Cancérologie & Antinéoplasiques \\
\hline & & Hématologie & Antinéoplasiques \\
\hline Ranibizumab & LUCENTIS & Ophtalmologie & $\begin{array}{l}\text { Dégénerescence maculaire liée ò l'âge et } \\
\text { oedème maculaire }\end{array}$ \\
\hline \multirow{3}{*}{ Rituximab } & \multirow{3}{*}{ MABTHERA } & Cancérologie & Antinéoplasiques \\
\hline & & Hématologie & Antinéoplasiques \\
\hline & & Rhumatologie & Immunosuppresseurs \\
\hline Tocilizumab & ROACTEMRA & Rhumatologie & Immunosuppresseurs \\
\hline \multirow{2}{*}{ Trastuzumab } & \multirow{2}{*}{ HERCEPTIN } & Cancérologie & Antinéoplasiques \\
\hline & & Hématologie & Antinéoplasiques \\
\hline
\end{tabular}

Tableau IV :

Anticorps monoclonaux commercialisés en France (Vidal 2012) 
I Les anti-TNFa se positionnent en deuxième intention, après échec du MTX (ou réponse insuffisante) pris à dose optimale tolérée pendant au moins trois mois ; ou bien en cas d'intolérance à celui-ci ; ou encore en cas d'échec à un autre traitement de fond ayant une efficacité structurale (léflunomide ou sulfasalazine), maintenu pendant au moins trois mois à dose optimale tolérée $[3,4]$.

I Les anti-TNFa sont utilisables en première intention dans une indication particulière : il s'agit des cas de $\mathrm{PR}$ active et sévère d'emblée, auxquels on peut proposer l'association MTX + anti-TNFa [3].

I Lutilisation des anti-TNFa est possible en monothérapie [3], par exemple en cas d'intolérance au MTX ou lorsque la poursuite du MTX est inadaptée, sauf en ce qui concerne l'infliximab (Remicade ${ }^{\varpi}$ ), qui dans la PR doit toujours être associé au MTX.

I Lanakinra est indiqué chez les patients en association au méthotrexate après échec d'au moins un traitement de fond. Mais son efficacité modérée par rapport aux anti-TNFa et son profil de tolérance le réservent plutôt aux malades ayant une contre-indication aux anti-TNFa [1].

I Le rituximab (Mabthera ${ }^{\circledR}$ et l'abatacept (Orencia ${ }^{\circledast}$ ) sont indiqués, en association au MTX, dans les PR sévères et évolutives, en cas de réponse insuffisante ou d'intolérance à d'autres traitements de fond, dont au moins un anti-TNFa. Mais la HAS recommande plutôt de tester deux anti-TNFa avant de passer au rituximab (Mabthera $\left.{ }^{\circledast}\right)$ ou à l'abatacept (Orencia ${ }^{\circledR}$ ) [1]

\ Le tocilizumab (Roactera ${ }^{\circledR}$ ) est indiqué (AMM européenne) en association au MTX, en cas de réponse inadéquate ou d'intolérance à un ou plusieurs traitements de fonds classiques ou à un ou plusieurs anti-TNFa.

Lefficacité d'une biothérapie dans la PR s'évalue après 12 semaines, sauf pour le rituximab (Mabthera ${ }^{\varpi}$ ), où l'on attend le quatrième mois ; de même pour l'abatacept (Orencia ${ }^{\circledR}$ ), où selon le contexte on peut prolonger le délai de surveillance de 12 à 24 semaines. Pour juger de cette efficacité, les rhumatologues se basent sur le DAS 28 (Disease Activity Score: indice composite tenant compte du nombre d'articulations gonflées et/ ou douloureuses, de la vitesse de sédimentation et de l'activité de la maladie évaluée par le patient sur une échelle analogique) et sur la réponse EULAR (déterminée selon l'évolution du DAS 28 entre deux mesures).

\section{Dans la spondylarthrite ankylosante (SA) et le rhumatisme psoriasique (RP)}

Seuls les anti-TNFa ont une indication parmi les biothérapies dans ces deux pathologies. À noter que les anti-TNFa sont volontiers utilisés seuls dans la SA et le RP, y compris l'infliximab (Remicade ${ }^{\varpi}$ ) [5].

I Pour les formes à prédominance axiale de SA et de RP, les anti-TNFa sont indiqués après échec des traitements conventionnels, c'est-à-dire après échec d'au moins 3 AINS, à la dose optimale tolérée, pendant au moins 3 mois. Rappelons que le MTX ne constitue pas un traitement de fond des formes axiales de SA et de RP [5].
I En présence d'une SA ou d'un RP à prédominance périphérique, les anti-TNFa ont leur place en cas d'échec à au moins un des traitements de fond suivants : MTX, léflunomide, sulfasalazine, utilisés pendant au moins 4 mois [5].

L’efficacité du traitement est évaluée à 12 semaines, grâce à l'indice BASDAI (Bath Ankylosing Spondylitis Disease Activity Index) dans les formes axiales et au nombre d'articulations gonflées et douloureuses dans les formes périphériques.

\section{Les autres indications (Vidal 2012)}

I En rhumatologie, deux anti-TNFa [étanercept $\left(\right.$ Enbrel $^{\circledR}$ ) et adalimumab (Humira $\left.{ }^{\circledR}\right)$ ] sont indiqués dans l'arthrite juvénile idiopathique polyarticulaire. Létanercept, dans sa forme pédiatrique, s'adresse aux enfants et adolescents de 4 à 17 ans en cas de réponse inadéquate ou d'intolérance au MTX. Ladalimumab est indiqué chez l'adolescent de 13 à 17 ans, en association au MTX, en cas de réponse insuffisante à un ou plusieurs traitements de fond, ou en monothérapie, en cas d'intolérance au MTX.

Une étude franco-américaine a étudié l'efficacité d'un nouveau traitement mensuel, anakinra (Kineret ${ }^{\circledR}$ prescrit à $2 \mathrm{mg}$ par kilo en sous-cutané) chez 24 enfants atteints d'arthrite juvénile systémique. Cette biothérapie agit sur un messager de l'inflammation (IL-1) à l'origine de la douleur et des destructions osseuses. La moitié des enfants ont bénéficié pendant un mois du traitement, l'autre moitié recevant un placebo. Après un mois, 8 des 12 patients sous Kineret ${ }^{\circledR}$ ont vu leur situation s'améliorer contre 1 sur 12 dans le groupe placebo. Durant les 11 mois de suivi, une proportion importante de patients ont pu arrêter ou réduire le recours aux corticoïdes.

I Larthrite juvénile idiopathique systémique, également connue sous le nom de maladie de Still, est une forme rare d'arthrite juvénile idiopathique (AJI). Cette maladie est difficile à diagnostiquer, en raison du manque de spécificité des premiers symptômes. La forme systémique se distingue des autres arthrites juvéniles idiopathiques par la présence d'une fièvre caractéristique qui évolue par pics suivis de franches chutes de température. Cette fièvre peut être associée à d'autres signes d'atteinte systémique (éruption cutanée, parfois péricardite).

On estime que $10 \%$ des jeunes patients atteints d'AJI (entre 2000 et 4000 ) présentent la forme systémique, dont la cause est inconnue. La maladie est le plus souvent diagnostiquée à lâge de 5 ans, mais il est fréquent que les manifestations apparaissent chez des enfants de 2-3 ans. En cas de doute, il est vivement conseillé au praticien d'adresser l'enfant à l'un des 18 centres de compétence, lesquels pourront se tourner vers l'un des deux centres de référence en rhumatologie pédiatrique. L'AJIs est la forme la plus destructrice des polyarthrites. Outre la douleur, qui fluctue au gré des pics de fièvre et des poussées inflammatoires, ce rhumatisme peut entraîner des séquelles à vie telles qu'un retard statural, 
un risque élevé d’ostéoporose précoce et un handicap. Une minorité ( 5 à $8 \%$ des jeunes patients) peut également voir son pronostic vital compromis, en raison d'une insuffisance hépatique. Enfin, le risque infectieux est également élevé.

Dans les premiers temps de la maladie, le traitement est essentiellement symptomatique et repose sur les anti-inflammatoires non-stéroïdiens. En cas de fièvre persistante et d'apparition d'une arthrite, la corticothérapie devient incontournable. Chez la moitié des patients, ce traitement s'avère suffisant. Mais chez les autres, la maladie évolue et l'organisme devient intolérant à la cortisone, laquelle ne bloque plus l'inflammation. On doit alors recourir à des immunosuppresseurs spécifiques, les anti-TNF [étanercept $\left(\right.$ Enbrel $\left.^{\circledR}\right)$, adalimubab (Humira ${ }^{\circledR}$ ), abatacept (Orencia ${ }^{\circledR}$ )] ou encore à une biothérapie agissant spécifiquement sur les interleukines-1 impliquées dans l'inflammation : anakinra (injection quotidienne), Rilanocept (injection hebdomadaire) ou canakinumab (injections toutes les 4 semaines, non douloureuses).

Le tocilizumab [Roactemra ${ }^{\circledast}$ cible spécifiquement l'interleukine 6 (IL 6)] : son rôle clé dans l’arthrite juvénile idiopathique a été démontré. Cette biothérapie est actuellement utilisée dans le traitement de la polyarthrite rhumatoïde active modérée à sévère de l'adulte en cas de réponse inadéquate ou d'intolérance à un précédent traitement. Depuis début août 2011, elle est également indiquée comme traitement de l'AJIs évolutive chez des patients âgés de 2 ans et plus résistants aux AINS et aux corticoïdes systémiques.

Létude menée pendant 12 semaines chez 112 enfants âgés de 2 à 17 ans montre qu'à l'inverse des autres biothérapies, le tocilizumab (Roactemra ${ }^{\circledR}$ ) permet d'obtenir des réponses plus constantes, et surtout que les injections sont nettement moins douloureuses (injections intraveineuses toutes les 2 semaines, contre injections sous-cutanées quotidiennes ou hebdomadaires selon les produits).

En France, le passage à la biothérapie intervient au bout de 6 mois et alors que le patient ne tolère plus la cortisone. Mais, aux Pays-Bas par exemple, il est d'usage de recourir à ce traitement dès 3 semaines d'évolution, conformément à la stratégie de "frapper vite et fort».

Il est encore trop tôt pour connaître la durée du traitement ainsi que les effets à long terme d'un tel traitement. Des marqueurs permettent d'ores et déjà de savoir si la rémission est suffisante.

I En dermatologie, trois anti-TNFa ont une AMM dans le psoriasis en plaques, en seconde intention après échec des autres traitements.

\En gastro-entérologie, l’adalimumab (Humira ${ }^{\circledR}$ ) et l'infliximab (Remicade ${ }^{\circledast}$ ) sont indiqués dans les maladies inflammatoires chroniques de l'intestin. Le premier chez les patients porteurs d'une maladie de Crohn n'ayant pas répondu à un traitement approprié par un corticoïde et/ou un immunosuppresseur, ou chez lesquels ce traitement est contre-indiqué ou mal toléré ; le second dans la même indication, ainsi que dans la maladie de Crohn fistulisée, également chez l'enfant de 6 à 17 ans ayant une maladie de Crohn sévère et active, et enfin en cas de rectocolite hémorragique chez les patients qui nont pas répondu à un traitement conventionnel ou lorsque ce traitement est mal toléré ou contre-indiqué $[6,10]$.

\ En hématologie, le rituximab (Mabthera ${ }^{\circledR}$ ) est indiqué en cas de lymphome.

\ En oncologie, le bévacizumab (Avsatin ${ }^{\circledR}$ ) est indiqué en cas de cancer colorectal métastatique, cancer du sein métastatique, du rein métastatique ou cancer bronchique non à petites cellules. La tumeur exprime des récepteurs particuliers pour les tumeurs digestives, on retrouve des antigènes comme l'ACE et EGFR qui sont des antigènes de reconnaissance spécifique pour les cancers. Lanticorps, une fois injecté, va pouvoir reconnaître l'antigène spécifique de la tumeur et se fixer dessus et non pas sur les tissus sains. Ainsi l'anticorps, fixé sur l'antigène, va pouvoir bloquer la progression des cellules tumorales et donc bloquer la croissance de la tumeur (fig. 1).

1 D'autres indications des anti-TNFa sont en cours d'évaluation : vascularites, sarcoïdose, lupus...

\section{EN PRATIQUE}

I Concernant les anti-TNFa, il n'y a aucune raison d'en choisir a priori un plutôt qu'un autre $[1,4]$. Le choix se fait au cas par cas, en tenant compte du mode de vie du malade, d'éventuelles comorbidités (MICI), et du terrain en général.

I Dans la PR, en cas d'échec à un premier anti-TNFa, la tendance actuelle est d'en tester un second avant de passer aux autres biothérapies ou à un autre protocole [1]. En revanche, il n'est pas recommandé d'essayer les trois produits de la classe, car la probabilité de réponse diminue au fur et à mesure des échecs et l'enjeu dans la PR est de ne pas perdre de temps.

\ Parmi les traitements de fond pouvant être associés aux anti-TNFa dans la PR, l'association avec le MTX est recommandée [1]. L'association avec des traitements de fond classiques (léflunomide, sulfasalazine en particulier) est possible [3], mais la HAS précise à ce propos que des études supplémentaires sont nécessaires [1]. Par ailleurs, on n'associe jamais deux anti-TNFa. Quant aux essais d'association des antiTNFa avec d'autres biothérapies, ils se sont soldés par une augmentation du taux dévénements infectieux.

1 S’agissant de la durée de traitement sous biothérapies, la situation diffère selon la pathologie causale. Dans certains cas de PR, lorsqu'une rémission complète de la maladie a été obtenue sur une période d'au moins 6 à 12 mois, on peut tenter un espacement progressif des doses, puis, si le contrôle de la maladie reste effectif, de suspendre le traitement de fond tout en poursuivant la surveillance. Il faut savoir repérer la fenêtre d'opportunité qui permet d'optimiser cette décroissance thérapeutique, à la condition d'avoir pris en charge le patient dès le début de sa maladie. Dans ces conditions, même si le risque de récidive 


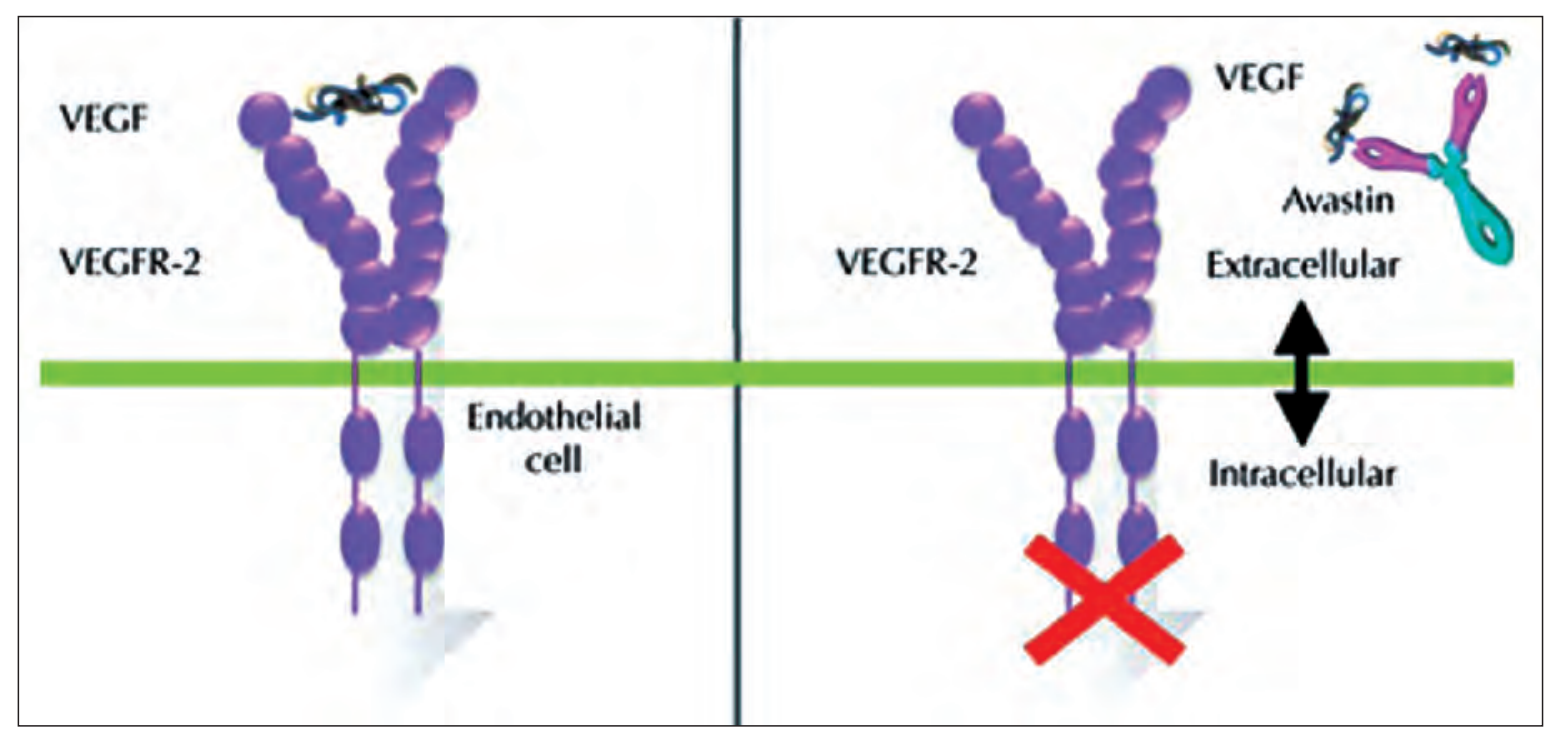

$\triangle$ Fig. 1 :

Mode d'action du bévacizumab. L'angiogénèse est provoquée par l'interaction entre VEGF et VEGFR-2. Le bévacizumab inhibe le VEGF extracellulaire et donc également l'angiogenèse au niveau de la tumeur.

n'est pas négligeable, les taux de rémission à un an approchent les 50 à $60 \%$. Une étude néerlandaise [11], l'étude Best, montre que parmi des PR récentes, ce sont celles qui sont traitées intensivement dès le départ (infliximab + un autre traitement de fond) qui ont à 4 ans le plus fort taux de contrôle de la maladie permettant l'arrêt de l'anti-TNF et la moindre progression des lésions radiologiques. Il n'est en est pas de même dans la SA chronique, où l'on observe $90 \%$ de rechute après l'arrêt des anti-TNFa.

\section{LES ÉLÉMENTS UTILES AU SUIVI}

\section{Biothérapies et risque infectieux}

Si la poursuite du traitement par anti-TNFa majore à des degrés divers le risque d'infection per- ou post-opératoire $[12,15]$, selon le contexte nosologique et la nature de la chirurgie, le risque de défaut ou de retard de cicatrisation reste peu probable. En effet, dans une étude ouverte japonaise durant laquelle le devenir de patients opérés d'une chirurgie orthopédique lourde (arthrodèse de cheville, prothèses de cheville, genoux, hanches, coudes, épaules) a été comparé selon qu'ils avaient été traités par anti-TNFa (39 patients) ou non (groupe contrôle de 74 patients), le taux de défaut de cicatrisation n'a été que dans $5,1 \%$ du groupe antiTNFa versus 6,8\% du groupe sans anti-TNFa [16], confortant les données d'une autre série japonaise [17]. En revanche, le risque d'infections per- ou postopératoires sous anti-TNFa a été, depuis les précédentes recommandations, réévalué, et plutôt à la hausse, par plusieurs études réalisées depuis 2007, tant dans le contexte des MICI, surtout la rectocolite ulcéro-hémorragique (RCUH), que dans celui des rhumatismes inflammatoires chroniques (RIC).

En résumé, le risque infectieux chez les rhumatismes inflammatoires chroniques (PR essentiellement) sous anti-TNFa qui se font opérer semble nettement accru dans certaines circonstances au moins :

I interventions sur les articulations superficielles où les téguments cicatrisent le plus mal (coudes, chevilles);

I pose de prothèse, et surtout reprise de prothèse;

I infection passée de la peau et/ou d'une cicatrice ;

I co-prise de prednisone, surtout à des posologies supérieures à $5 \mathrm{mg} /$ jour ;

$\checkmark$ autre cause d'immunosuppression associée.

Ces paramètres ainsi que lâge des patients doivent être pris en compte pour évaluer, au cas par cas, le délai nécessaire d'arrêt des anti-TNFa avant la chirurgie $[18,24]$. La présence de plusieurs de ces co-facteurs de risque devrait faire évoquer un risque infectieux élevé, voire très élevé en cas de pose ou dépose de prothèses associée.

v Chez un patient recevant une biothérapie, la surveillance du risque infectieux est un élément essentiel du suivi. Selon une méta-analyse parue en 2006 [25], ce risque est multiplié par deux avec les anti-TNFa. D’autres sources donnent des chiffres légèrement supérieurs, mais il faut savoir que l'augmentation du risque infectieux concerne toutes les biothérapies et ne se limite pas aux seuls anti-TNFa. Ce surrisque est surtout présent la première année de traitement. Par la suite, lorsque la maladie inflammatoire est contrôlée, le risque infectieux diminue.

\ Le suivi en médecine générale doit donc s'attacher à repérer la survenue d'une infection, en tenant compte des antécédents du patient. La présence d'une fièvre constitue toujours un signe d'alerte et doit faire rechercher une complication infectieuse. En présence de signes de gravité, l'anti-TNFa est arrêté et le patient hospitalisé. Dans les autres situations, c'est la présence d'un signe de localisation qui guide la conduite à tenir : ECBU en urgence et traitement immédiat en cas de symptômes urinaires, antibiothérapie en cas d'angine ou de bronchite simple. L'idéal est de réaliser les prélèvements nécessaires au diagnostic. Larrêt de 
l'anti-TNFa est systématique, le traitement pouvant être repris après guérison de l'épisode infectieux. Des fiches pratiques éditées par le CRI [26] indiquent les principaux éléments à rechercher chez un patient traité par anti-TNFa. De façon générale, la HAS [3] préconise de prendre un avis spécialisé.

ע Les complications infectieuses sous anti-TNF peuvent être bactériennes ou virales : pneumopathies, infections urinaires ou cutanées, réveil d'une tuberculose latente. Concernant ce dernier point, l'Afssaps a édicté des recommandations [27]. La philosophie générale est d'évaluer (interrogatoire, examen clinique, IDR, radiographie thoracique) pour chaque patient candidat à un traitement par anti-TNFa le risque de tuberculose latente ou active. Si une tuberculose latente est dépistée, un traitement prophylactique par rifampicine + isoniazide doit être instauré pendant 3 mois (d'autres schémas sont possibles), et la première injection d'anti-TNFa doit être différée de 3 semaines. En cas de tuberculose active diagnostiquée initialement ou survenant sous anti-TNFa, une quadrithérapie (rifampicine, isoniazide, pyrazinamide, éthambutol) est mise en place pendant 2 mois, relayée par une bithérapie (rifampicine + isoniazide). La durée totale du traitement antituberculeux varie entre 6 et 12 mois selon la localisation. Selon le contexte et le bénéfice escompté sous biothérapie, l'anti-TNFa pourra être repris avant la fin du traitement antituberculeux.

\section{LE RISQUE DE CANCER}

Les données concernant l'augmentation du risque néoplasique sous anti-TNFa ne sont pas homogènes. La méta-analyse de Bongartz [25] fait état d'un risque multiplié par 3,3 tandis que la HAS précise qu'il n’a pas été mis en évidence d'augmentation du risque de développer une tumeur solide [1]. Il est vrai que les résultats diffèrent selon les registres de surveillance considérés. Deux points semblent cependant se confirmer. Le premier concerne l'augmentation du risque de lymphome dans les populations de PR traitées par anti-TNFa, par rapport aux patients traités de manière conventionnelle. Pour autant, l'imputabilité des anti-TNFa n'est pas certaine ; en effet, l'existence d'une PR est de fait associée à un doublement du risque de survenue d'un lymphome (versus la population générale), variable en fonction de l'intensité de l'inflammation. Les antiTNFa étant réservés aux PR les plus sévères et les plus actives, il y a peut-être là un biais dont il faut tenir compte. Ce point est encore discuté actuellement. Les derniers résultats obtenus par l'observatoire RATIO, qui recense les effets indésirables des anti-TNFa, font état d'un risque de lymphome plus élevé sous infliximab (Remicade ${ }^{\circledast}$ ) ou adalimumab (Humira ${ }^{\circledR}$ ) que sous étanercept $\left(\right.$ Enbrel $\left.^{\circledR}\right)$. Le deuxième aspect concerne l'augmentation du risque de cancers cutanés, notamment au cours de la première année de traitement. Il s'agit essentiellement de cancers basocellulaires et spinocellulaires, mais des mélanomes ont également été observés.

\section{LES AUTRES EFFETS INDÉSIRABLES}

La fonction cardiaque (risque d'insuffisance cardiaque) et l'état neurologique (démyélinisation) doivent également être surveillés sous anti-TNFa.

Pour le rituximab (Mabthera $\left.{ }^{\circledR}\right)$, et à un moindre degré pour l'abatacept (Orencia ${ }^{\circledR}$ ), outre le risque infectieux déjà cité, le principal effet indésirable est la survenue d'une réaction générale au moment de la perfusion.

\section{CHIRURGIE PROGRAMMÉE}

Le délai d’arrêt doit être d'au moins deux semaines pour l'étanercept $\left(\right.$ Enbrel $\left.^{\circledR}\right)$ et d'au moins 4 semaines pour l'infliximab (Remicade ${ }^{\circledast}$ ) et l'adalimumab (Humira ${ }^{\circledR}$ ), s'il n'y a pas de circonstances à risques [28]. Il doit en fait souvent être plus important (jusqu'à 4 ou 5 demivies : voir tableau $\mathbf{V}$ pour l'estimation par molécule) en fonction :

I du risque infectieux lié à la chirurgie : faible pour les chirurgies en «milieu stérile» (exemple : cataracte), élevé pour les chirurgies en "milieu septique» (exemple : sigmoïdite) ou les situations à «risque septique» (exemple : prothèse articulaire, surtout s'il s'agit d'une reprise chirurgicale);

I du terrain et du risque infectieux propre au patient : antécédents infectieux, en particulier infection passée de la peau et/ou d'une cicatrice, existence de prothèses articulaires, d'un diabète, d'une corticothérapie associée;

I de la sévérité de l’affection rhumatologique et de son contrôle par le traitement : un délai d’arrêt plus pro-

\begin{tabular}{|l|l|l|l|l|l|}
\hline \multicolumn{2}{|l|}{ Risque septique per-opératoire : } & Farible & Moyen & Elevé & Très élevé \\
\hline Molécule & Demi-vie & $\mathbf{2}$ demi-vies & $\mathbf{3}$ demi-vies & 4 demi-vies & $\mathbf{5}$ demi-vies \\
\hline Etanercept & $70 \mathrm{~h}$ & $10 \mathrm{j} \sim 2 \mathrm{sem}$ & $15 \mathrm{j} \sim 2 \mathrm{sem}$ & $20 \mathrm{j} \sim 3 \mathrm{sem}$ & $25 \mathrm{j} \sim 4 \mathrm{sem}$ \\
\hline Infliximab & $10 \mathrm{j}$ & $20 \mathrm{j} \sim 3 \mathrm{sem}$ & $30 \mathrm{j} \sim 4 \mathrm{sem}$ & $40 \mathrm{j} \sim 6 \mathrm{sem}$ & $50 \mathrm{j} \sim 8 \mathrm{sem}$ \\
\hline Certolizumab & $10-15 \mathrm{j}$ & $30 \mathrm{j} \sim 4 \mathrm{sem}$ & $45 \mathrm{j} \sim 6 \mathrm{sem}$ & $60 \mathrm{j} \sim 8 \mathrm{sem}$ & $75 \mathrm{j} \sim 10 \mathrm{sem}$ \\
\hline Golimumab & $10-15 \mathrm{j}$ & $30 \mathrm{j} \sim 4 \mathrm{sem}$ & $45 \mathrm{j} \sim 6 \mathrm{sem}$ & $60 \mathrm{j} \sim 8 \mathrm{sem}$ & $75 \mathrm{j} \sim 10 \mathrm{sem}$ \\
\hline Bélatacept & $10-15 \mathrm{j}$ & $30 \mathrm{j} \sim 4 \mathrm{sem}$ & $45 \mathrm{j} \sim 6 \mathrm{sem}$ & $60 \mathrm{j} \sim 8 \mathrm{sem}$ & $75 \mathrm{j} \sim 10 \mathrm{sem}$ \\
\hline
\end{tabular}

Tableau V: Durées d’arrêt préalables des anti-TNF $\alpha$ conseillées avant intervention chirurgicale 
longé, diminuant probablement le risque infectieux postopératoire, n'expose pas toujours le patient à une recrudescence de sa maladie, un rebond n'ayant par exemple été observé dans la période péri-opératoire que dans $17 \%$ des cas dans l'étude de Kawakami et al. [17]. Dans tous les cas, la reprise du traitement par anti-TNFa ne sera autorisée qu'après cicatrisation complète et en l'absence d'infection, et avec une prudence toute particulière en cas de reprise de prothèse. Une étude du taux sérique détanercept (pris à $50 \mathrm{mg}$ par semaine depuis plusieurs mois) chez sept patients japonais devant être opérés a été réalisée à 1 , $4,7,11,14$ et 21 jours après l'arrêt du traitement [29]. Les auteurs ont estimé que le traitement pourrait, compte tenu des taux mesurés, être repris 10 jours après l'arrêt du traitement en l'absence de sepsis. Ce délai pourrait être en fait arrondi à deux semaines car seulement deux des sept patients avaient connu une poussée de leur PR dans les 14 jours suivant l'arrêt de ce traitement [29].

\section{CHIRURGIE EN URGENCE}

Lorsque le geste chirurgical ne peut être différé, les recommandations d'experts sont :

$\checkmark$ arrêt du traitement par anti-TNFa ;

1 discuter une antibiothérapie prophylactique de couverture en cas de chirurgie à risque septique (exemple : péritonite) [30] ;

I surveillance rigoureuse postopératoire ;

I reprise du traitement autorisée uniquement après cicatrisation (et arrêt d'une éventuelle antibiothérapie) et en l'absence d'infection.

\section{Recommandations des Sociétés de rhumatologie}

Les recommandations françaises de 2005 [23] étaient un délai minimum d'arrêt de 2 semaines pour l'étanercept $\left(\right.$ Enbrel $\left.^{\circledR}\right)$ et 4 semaines pour l'adalimumab (Humira ${ }^{\circledR}$ ) et l’infliximab (Remicade ${ }^{\circledR}$ ), ce délai pouvant être majoré en cas de chirurgie à risque.

Celles encore en vigueur aux Pays-Bas (mais datant de 2003) conseillent de respecter un délai de 4 demivies [31].

Les recommandations de la BSR (British Society of Rheumatology) proposaient, en juillet 2004, d'interrompre les anti-TNFa, sans distinction entre les produits, 2 à 4 semaines avant toute intervention chirurgicale «majeure», et de reprendre le traitement après cicatrisation, en l'absence de signes d'infection [32].

Dans l'actualisation de septembre 2010 [33], il est proposé de moduler la nécessité de l’arrêt et de sa durée en fonction du type de chirurgie, et du risque de rebond de la PR, mais d'interrompre l'anti-TNFa dans un délai de 3 à 5 fois la demi-vie de cette molécule pour les chirurgies à risque significatif d'infection (soit un durcissement des recommandations par rapport au délai préalablement suggéré de seulement deux à quatre semaines).

\section{Synthèse des différentes recommandations}

Au final, et en retenant pour l'estimation de la demivie les valeurs moyennes rapportées dans la littérature pour chaque molécule, les durées d'arrêt préalables des anti-TNFa conseillées sont résumées dans le tableau V. Par ailleurs, les traitements par anti-TNFa ne devraient être repris que deux semaines au moins après une chirurgie à risque élevé d'infection, et seulement en cas de cicatrisation complète et en l'absence d'infection [28].

\section{SOINS DENTAIRES}

Il est recommandé une hygiène bucco-dentaire et des soins réguliers, ainsi que la réalisation d'un panoramique dentaire au moindre doute.

En cas d'état bucco-dentaire défectueux, des soins appropriés devront être effectués avant de débuter un traitement par anti-TNFa.

Ces soins devraient concerner surtout le parodonte, et motiver donc l'avis d'un chirurgien dentiste, d'autant que plusieurs études ont suggéré que la prise en charge correcte de la maladie parodontale réduit l'activité de la PR (34). De plus, le seul travail ayant étudié de manière prospective le statut parodontal des patients traités par anti-TNFa a conclu que les anti-TNFa n'accéléraient pas l'alvéolyse mais majoraient l'inflammation gingivale [35].

I Soins usuels (caries, détartrage) : il n’y a pas d’éléments justifiant l'arrêt du traitement.

I Soins à risque infectieux (avulsions, granulome apical, abcès...) : il est recommandé un arrêt du tocilizumab (Roactemra ${ }^{\circledast}$ ) avant soins dentaires, d'au moins 4 semaines et de proposer une antibioprophylaxie [36].

I Implants : il n'y a pas d'indication formelle à arrêter le tocilizumab (Roactemra ${ }^{\circledR}$ ), tout en restant vigilant aux risques infectieux potentiels.

\section{CONCLUSION}

Les anti-TNFa sont devenus un traitement essentiel des MICI, tant dans l'obtention de la rémission sans corticoïde que dans son maintien. Leur prescription justifie un bilan préalable rigoureux et idéalement une prescription concertée, à l'instar des chimiothérapies anti-cancéreuses, dans la mesure où l'on engage des patients souvent jeunes sur des traitements au long cours. Globalement, le taux d'effet indésirable sous anti-TNFa semble proche de celui des corticoïdes et des immunosuppresseurs. Selon l'expérience danoise, $47 \%$ des malades traités par infliximab ont développé au moins un effet indésirable qui était considéré comme sévère dans $15 \%$ des cas [37]. Il peut alors s'agir d'infections graves ou de manifestations paradoxales qu'il faut savoir reconnaître bien qu'elles dépassent le domaine de la gastroentérologie.

Les anticorps monoclonaux et les protéines de fusion 
actuellement sur le marché ont conduit au cours de la dernière décennie à des résultats cliniques encourageants, principalement lorsqu'ils ont été utilisés en combinaison avec d'autres molécules issues de la recherche pharmaceutique, comme le méthotrexate, la fludarabine (Fludara ${ }^{\circledR}$ ), ou l'irinotecan (CamptoW ${ }^{\circledast}$ ) par exemple. Les efforts se portent désormais sur la conception d'anticorps de seconde et troisième génération, bénéficiant des connaissances récemment acquises sur la structure des anticorps, leurs interactions avec l'antigène, la fixation au C1q et aux différents RFcg activateurs et inhibiteurs, leur demivie et leur biodistribution. Les objectifs principaux de ces efforts sont évidemment d'accroître leur efficacité, de mieux contrôler les effets indésirables qu'ils provoquent, mais aussi d'abaisser le coût des traitements par patient (allant actuellement de quelques milliers d'euros jusqu'à 50000 euros par an), en diminuant les doses requises tout en atteignant une bonne efficacité thérapeutique [2].

Les prochaines années nous diront donc si l'optimisation des propriétés effectrices des anticorps permet effectivement lobtention de meilleures réponses cliniques, sans induire d'effets secondaires plus importants. Si c’était le cas, cela représenterait un grand succès de l'immunothérapie ciblée et, plus généralement, des biothérapies, et un encouragement à explorer de nouveaux champs cliniques à l'aide de telles approches.

\section{Bibliographie}

[1] Haute Autorité de Santé. Recommandations professionnelles. Polyarthrite rhumatoïde. Prise en charge en phase d'état. 2007.

[2] Teillaud JL. Qu’est ce qu'une biothérapie? L'exemple des anticorps monoclonaux. Presse Med. 2009;38:825-31.

[3] Haute autorité de Santé. Polyarthrite rhumatoïde. Synthèses des recommandations. Septembre 2007.

[4] Fautrel B, Pham T, Mouterde G, Le Loët X, Goupille P, Guillemin F, Ravaud P, Cantagrel A, Dougados M, Puéchal X, Sibilia J, Soubrier M, Mariette X, Combe B. Club Rhumatismes et Inflammation; Société Française de Rhumatologie. Recommendations of the French Society for Rheumatology regarding TNFalpha antagonist therapy in patients with rheumatoid arthritis. Joint Bone Spine. 2007;74(6):627-37.

[5] Hession MT, Gottlieb AB. Perioperative management of tumor necrosis factor antagonists in patients with psoriasis and other inflammatory disorders. J Dermatolog Treat. 2011;22(2):90-101.

[6] Marchal L, D’Haens G, van Assche G, et al. The risk of post-operative complications associated with infliximab therapy for Crohn's disease : a controlled cohort study. Aliment Pharmacol Ther 2004;19:749-54.

[7] Colombel JF, Loftus EV Jr, Tremaine WJ, et al. Early postoperative complications are not increased in patients with Crohn's disease treated perioperatively with infliximab or immunosuppressive therapy.

Am J Gastroenterol 2004;99:878-83.
[8] Kunitake H, Hodin R, Shellito PC, et al. Perioperative treatment with infliximab in patients with Crohn's disease and ulcerative colitis is not associated with an increased rate of postoperative complications.

J Gastrointest Surg 2008;12:1730-6.

[9] Appau KA, Fazio VW, Shen B, et al. Use of infliximab within 3 months of ileocolonic resection is associated with adverse postoperative outcomes in Crohn's patients. J Gastrointest Surg 2008;12:1738-44

[10] Yang Z, Wu K, Fan D. Meta-analysis: preoperative infliximab treatment and short-term post-operative complications in patients with ulcerative colitis. Aliment Pharmacol Ther 2010;31:486-92.

[11] Van der Kooij SM, le Cessie S, Goekoop-Ruiterman YP, de Vries-Bouwstra JK, van Zeben D, Kerstens PJ, Hazes JM, van Schaardenburg D, Breedveld FC, Dijkmans BA, Allaart CF. Clinical and radiological efficacy of initial versus delayed treatment with infliximab plus methotrexate in patients with early rheumatoid arthritis.

Ann Rheum Dis. 2009;68(7):1153-8.

[12] Selvasekar CR, Cima RR, Larson DW, et al. Effect of infliximab on short-term complications in patients undergoing operation for chronic ulcerative colitis. J Am Coll Surg 2007;204:956-62.

[13] Mor IJ, Vogel JD, da Luz Moreira A, et al. Infliximab in ulcerative colitis is associated with an increased risk of postoperative complications after restorative proctocolectomy. Dis Colon Rectum 2008;51:1202-7.

[14] Coquet-Renier B, Berdah SV, Grimaud JC, et al. Preoperative infliximab treatment and postoperative complications after laparoscopic restorative proctocolectomy with ileal pouch-anal anastomosis: a casematched study. Surg Endosc 2010;24:1866-71.

[15] Regadas FS, Pinto RA, Murad-Regadas SM, et al. Short-term outcome of infliximab and other medications on patients with inflammatory bowel disease undergoing ileostomy reversal. Colorectal Dis. 2011;13(5):555-60.

[16] Hirano Y, Kojima T, Kanavama Y, et al. Influences of anti-tumour necrosis factor agents on postoperative recovery in patients with rheumatoid arthritis. Clin Rheumatol 2010;29:495-500.

[17] Kawakami K, Ikari K, Kawamura K, et al. Complications and features after joint surgery in rheumatoid arthritis patients treated with tumour necrosis factor-alpha blockers: perioperative interruption of tumour necrosis factor-alpha blockers decreases complications? Rheumatology (Oxford) 2010;49:341-7.

[18] Bibbo C, Goldberg JW. Infectious and healing complications after elective orthopaedic foot and ankle surgery during tumor necrosis factor-alpha inhibition therapy. Foot Ankle Int 2004;25:331-5.

[19] Wendling D, Balblanc JC, Brousse A, et al. Surgery in patients receiving anti-tumour necrosis factor alpha treatment in rheumatoid arthritis: an observational study on $\mathbf{5 0}$ surgical procedures. Ann Rheum Dis 2005;64:1378-9.

[20] Giles JT, Bartlett SJ, Gelber AC, et al. Tumor necrosis factor inhibitor therapy and risk of serious postoperative orthopedic infection in rheumatoid arthritis. Arthritis Rheum 2006;55:333-7.

[21] Matthews JLK, Martin L, Hu R. Postoperative complications in rheumatoid 
arthritis patients on anti-TNF therapies undergoing orthopaedic procedures. Ann Rheum Dis 2006;65(Suppl.II):331(Abs FRI0147).

[22] Salliot C, Gossec L, Ruyssen-Wiltrand A, et al. Infections during tumour necrosis factor-alpha blocker therapy for rheumatic diseases in daily practice : a systematic retrospective study of 709 patients. Rheumatology (Oxford) 2007;46:327-34.

[23] Ruyssen-Witrand A, Gossec L, Salliot C, et al. Complication rates of 127 surgical procedures performed in rheumatic patients receiving tumor necrosis factor alpha blockers. Clin Exp Rheumatol 2007;25:430-6.

[24] Gilson M, Gossec M, Mariette X, et al. Risk factors of total joint arthroplasty infection in patients receiving TNFa blockers : a case-control study. Arthritis Res Ther. 2010;12(4):R145.

[25] Bongartz T, Sutton AJ, Sweeting MJ, Buchan I, Matteson EL, Montori V. Anti-TNF antibody therapy in rheumatoid arthritis and the risk of serious infections and malignancies: systematic review and meta-analysis of rare harmful effects in randomized controlled trials. JAMA 2006;295(19):2275-85.

[26] Club Rhumatismes et Inflammation (CRI). Anti-TNF alpha et suivi de la tolérance. Fiches pratiques. Décembre 2006.
[27] AFSSAPS. Recommandations nationales. Prévention et prise en charge des tuberculoses survenant sous anti-TNF alpha. Juillet 2005.

[28] Société française de rhumatologie. Traitements anti-TNFa et suivi de la tolérance. Conduite à tenir en cas de chirurgie et soins dentaires. 2008.

[29] Nishida K, Hashizume K, Kadota Y, et al. Time-concentration profile of serum etanercept in Japanese patients with rheumatoid arthritis after treatment discontinuation before orthopedic surgery. Mod Rheumatol. 2010;20(6):637-9.

[30] Martin C, Bantz P, Thomachot L. Antibioprophylaxie en chirurgie. In Bergogne-Berezin E, Dellamonica P. Antibiothérapie en pratique clinique. Abrégés Masson, 2ème édition, Paris 1999.

[31] Den Broeder AA, Creemers MC, Fransen J, et al. Risk factors for surgical site infections and other complications in elective surgery in patients with rheumatoid arthritis with special attention for anti-tumor necrosis factor: a large retrospective study. J Rheumatol 2007;34:689-95.

[32] Ledingham J, Deighton C, British Society for Rheumatology Standards, Guidelines and Audit Working Group. Update on the British Society for Rheumatology guidelines for prescribing TNF $\alpha$ blockers in adults with rheumatoid arthritis (update of previous guidelines of April 2001). Rheumatology 2005;44:157-63.

[33] Ding T, Ledingham J, Luqmani R, et al. BSR and BHPR rheumatoid arthritis guidelines on safety of anti-TNF therapies. Rheumatology (Oxford) 2010;49(11):2217-9.

[34] Ortiz P, Bissada NF, Palomo L, et al. Periodontal therapy reduces the severity of active rheumatoid arthritis in patients treated with or without tumor necrosis factor inhibitors.

J Periodontol 2009;80:535-40.

[35] Pers JO, Saraux A, Pierre R, et al. AntiTNF-alpha immunotherapy is associated with increased gingival inflammation without clinical attachment loss in subjects with rheumatoid arthritis. J Periodontol 2008;79:1645-51.

[36] Tong DC, Rothwell BR. Antibiotic prophylaxis in dentistry : a review and practice recommendations. JADA 2000;131:366-74.

[37] Caspersen S, Elkjaer M, Riis L, Pedersen N, Mortensen C, Jess T, Sarto P, Hansen TS, Wewer V, Bendtsen F, Moesgaard F, Munkholm P. Infliximab for inflammatory bowel disease in Denmark 1999-2005: clinical outcome and follow-up evaluation of malignancy and mortality. Clin Gastroenterol Hepatol 2008;6:1212-7; quiz 1176. les Actualités Odonto-Stomatologiques?

Pour publier votre article original et être lu par l'ensemble des praticiens français, soumettez-le au comité de rédaction des AOS Téléchargez les instructions aux auteurs sur www.aos-journal.org Envoyez votre article à secrétariat.aos@edpsciences.org

\section{dans}

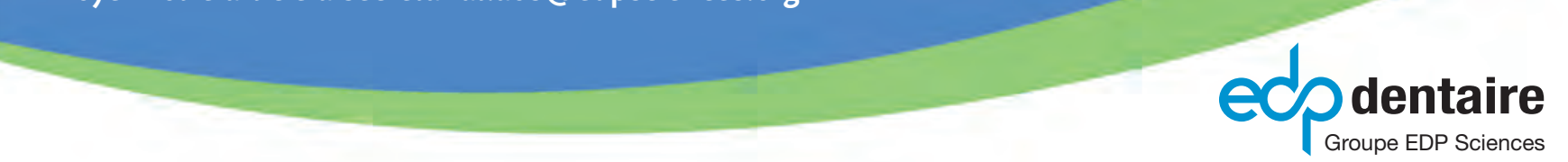

